\title{
Protein-bound polysaccharide-K induces apoptosis via mitochondria and p38 mitogen-activated protein kinase-dependent pathways in HL-60 promyelomonocytic leukemia cells
}

\author{
NORIYUKI HIRAHARA ${ }^{1}$, TAKEO EDAMATSU ${ }^{2}$, AYAKO FUJIEDA ${ }^{2}$, \\ MASAKI FUJIOKA $^{2}$, TSUTOMU WADA ${ }^{2}$ and YOSHITSUGU TAJIMA ${ }^{1}$ \\ ${ }^{1}$ Department of Digestive and General Surgery, Shimane University Faculty of Medicine, Izumo, \\ Shimane 693-8501; ${ }^{2}$ Biomedical Research Laboratories, Kureha Corporation, Tokyo 169-8503, Japan
}

Received January 18, 2013; Accepted March 15, 2013

DOI: $10.3892 /$ or.2013.2412

\begin{abstract}
Protein-bound polysaccharide-K (PSK) is extracted from Coriolus versicolor (CM101). PSK is a biological response modifier (BRM), and its mechanism of action is partly mediated by modulating host immune systems; however, recent studies showed antiproliferative activity of PSK. Therefore, we examined the mechanism underlying the antiproliferative activity of PSK using seven different human malignant cell lines (WiDr, HT29, SW480, KATOIII, AGS, HL-60 and U937), and PSK was found to inhibit the proliferation of HL-60 cells most profoundly. Therefore, HL-60 cells were used to elucidate the mechanism of the antiproliferative activity. Western blotting was performed to detect phosphorylated p38 mitogen-activated protein kinase (MAPK). A p38 MAPK inhibitor, SB203580, was used to examine the roles in PSK-induced apoptosis and growth inhibition. Flow cytometry was performed for mitochondrial membrane potential detection. PSK activated caspase- 3 and induced p38 MAPK phosphorylation. Co-treatment with SB203580 blocked PSK-induced apoptosis, caspase-3 activation and growth inhibition. PSK induced apoptosis via the mitochondrial pathway. The depolarization of mitochondria induced by PSK was reversed by co-treatment with SB203580. The present study revealed that PSK induced apoptosis in HL-60 cells via a mitochondrial and p38 MAPK-dependent pathway.
\end{abstract}

\section{Introduction}

Protein-bound polysaccharide-K (PSK) is a protein-bound polysaccharide K (Krestin), extracted from cultured mycelia of Coriolus versicolor (CM101) and the average molecular

Correspondence to: Dr Noriyuki Hirahara, Department of Digestive and General Surgery, Shimane University Faculty of Medicine, 89-1 Enya-cho, Izumo, Shimane 693-8501, Japan E-mail: norinorihirahara@yahoo.co.jp

Key words: protein-bound polysaccharide-K, apoptosis, mitochondrial pathway, p38 mitogen-activated protein kinase, HL-60 cell weight is approximately 100,000 (1). PSK is one of the biological response modifiers (BRM), and is used clinically in combination therapy for gastrointestinal cancer and small cell lung carcinoma (1-4).

The most important and widely reported property is the immunomodulatory effect, as direct antitumor activity is weak. A few previous reports have indicated that PSK induces cytostatic effects on growth and invasion by modulating the expression of major histocompatibility complex (MHC) class I and inhibition of nuclear factor kappaB (NF-кB) (5).

Our previous study demonstrated that PSK inhibits cellular proliferation in a cell type-specific manner and that the inhibition is most profound for promyelomonocytic leukemia HL-60 cells (6). We have shown that the underlying mechanism of the inhibition in cellular proliferation is due, in part, to caspase3-mediated apoptosis.

Apoptosis is one of the main mechanisms for inhibition of cancer growth and proliferation. The execution of apoptosis, or programmed cell death, is associated with characteristic morphological and biochemical changes mediated by a series of gene regulations and cell-signaling pathways (7). Introduction of apoptosis included activation of caspase, change of mitochondrial signaling pathway and regulation of Bcl-2 family members. Recently, perturbation of mitochondrial function has been shown to be a key event in the apoptotic cascade. Anticancer drugs may damage the mitochondria by increasing the permeability of the outer mitochondrial membrane, which is associated with the collapse of the mitochondrial membrane potential $(\Delta \Psi \mathrm{m})(8)$.

Therefore, the present study investigated the mechanism of the apoptosis induced by PSK and focused on the mitochondrial and p38 mitogen-activated protein kinase (MAPK)-dependent pathway in HL-60 cells.

\section{Materials and methods}

Cells. The promyelomonocytic leukemia cell line HL-60 was obtained from the American Type Culture Collection (ATCC, Manassas, VA, USA), and maintained in RPMI-1640 (Gibco-BRL, Rockville, MD, USA) supplemented with $10 \%$ fetal bovine serum (Life Technologies, Milan, Italy). 
Reagents. PSK was manufactured at Kureha Corp. (Tokyo, Japan), and was dissolved in Dulbecco's phosphate-buffered saline (DPBS) at a concentration of $100 \mathrm{mg} / \mathrm{ml}$ (Gibco-BRL). In each experiment, the PSK solution was freshly prepared and further diluted with medium. The p38 MAPK inhibitor SB203580 was purchased from LC Laboratories (Woburn, MA, USA), and dissolved in dimethyl sulfoxide (DMSO) at $100 \mathrm{mmol} / \mathrm{l}$ to prepare a stock solution. Prior to use, the stock solution was diluted with medium. The final concentration of SB203580 was $30 \mu \mathrm{mol} / 1$ and the final concentration of DMSO was $<0.1 \%$.

Western blot analysis. HL-60 cells were suspended in medium containing DPBS (control) or PSK $(100 \mu \mathrm{g} / \mathrm{ml})$, and seeded into $100-\mathrm{mm}$ culture dishes at a density of $3 \times 10^{5}$ cells/dish. After the indicated time of incubation, cells were harvested and lysed in RIPA Lysis Buffer (Santa Cruz Biotechnology, Santa Cruz, CA, USA) by rotating for $30 \mathrm{~min}$ at $4^{\circ} \mathrm{C}$. Following cell lysis, a clear cell lysate was obtained by centrifugation and the protein concentration was determined. Twenty micrograms of protein were used for western blotting. Antibodies against phosphorylated p38 MAPK and p38 MAPK (Cell Signaling Technology, Danvers, MA, USA) were used to detect phosphorylated form of p38 MAPK and total p38 MAPK, respectively. Chemiluminescent signals generated by using the ECL Advance ${ }^{\mathrm{TM}}$ Western Blotting Detection kit (GE Healthcare, Buckinghamshire, UK) were detected by LightCapture II Cooled CCD Camera systems (ATTO, Tokyo, Japan).

Evaluation of apoptosis. Phosphatidylserine externalization and membrane integrity were evaluated using TACS ${ }^{\mathrm{TM}}$ Annexin V-FITC Apoptosis Detection kit (Trevigen, Gaithersburg, MD, USA), which contains fluorescein isothiocyanate (FITC)-conjugated Annexin V (Annexin V-FITC) and propidium iodide (PI). HL-60 cells were suspended in medium containing DPBS (control) or PSK $(100 \mu \mathrm{g} / \mathrm{ml})$ in the presence or absence of SB203580 $(30 \mu \mathrm{mol} / \mathrm{l})$ and seeded into 6 -well plates at a density of $6 \times 10^{4}$ cells/well. After the indicated time of incubation, cells were harvested and stained using the kit, according to the manufacturer's instructions. Stained cells were analyzed by flow cytometry (FACSCalibur; Becton-Dickinson, Franklin Lakes, NJ, USA).

Detection of active caspase-3. HL-60 cells were suspended in medium containing DPBS (control) or PSK $(100 \mu \mathrm{g} / \mathrm{ml})$ in the presence or absence of SB203580 (30 $\mu \mathrm{mol} / \mathrm{l})$ and seeded into $175-\mathrm{cm}^{2}$ culture flasks at a density of $1.05 \times 10^{6}$ cells/flask. After the indicated time of incubation, cells were harvested and resuspended in PBS containing $1 \%$ saponin at a density of $1 \times 10^{6}$ cells $/ \mathrm{ml}$. The active form of caspase- 3 was detected using APO ACTIVE $3^{\mathrm{TM}}$ (antibody to active caspase-3; Cell Technology Inc., Minneapolis, MN, USA) according to the manufacturer's instructions. Stained cells were analyzed by flow cytometry.

Cellular proliferation assay. Cellular proliferation was determined using WST-8 (Dojindo, Kumamoto, Japan), a water-soluble form of methyl thiazolyl tetrazolium (MTT),

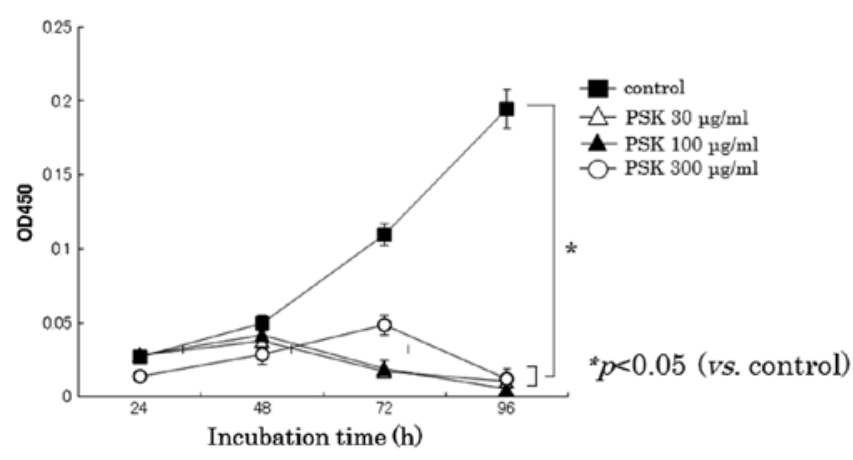

Figure 1. Effect of PSK on HL-60 proliferation. The HL-60 cell line was exposed to PSK or DPBS (control). Each time-point represents the means \pm SD $(\mathrm{n}=5)$. Statistical significance, ${ }^{*} \mathrm{P}<0.05$.

according to the manufacturer's instructions. Briefly, cells were suspended in medium containing DPBS (control) or PSK $(100 \mu \mathrm{g} / \mathrm{ml})$ in the presence or absence of SB203580 (30 $\mu \mathrm{mol} / \mathrm{l})$ and seeded into 96 -well plates at a density of $3 \times 10^{3}$ cells/well. After $72 \mathrm{~h}$ of incubation, WST- 8 was added to each well and incubated for another $3 \mathrm{~h}$. WST- 8 is reduced to an orange colored formazan, which has maximum absorption at $460 \mathrm{~nm}$. Optical density (OD) at $450 \mathrm{~nm}$ and $630 \mathrm{~nm}$ was measured. The OD at $630 \mathrm{~nm}$ was then subtracted from the $\mathrm{OD}$ at $450 \mathrm{~nm}$.

Flow cytometry for $\Delta \Psi m$ detection. The $\Delta \Psi \mathrm{m}$ of HL-60 cells after treatment with PSK $(100 \mu \mathrm{g} / \mathrm{ml})$ in the presence or absence of SB203580 (30 $\mu \mathrm{mol} / \mathrm{l})$ after the indicated time of incubation was measured using a Flow Cytometry Mitochondrial Membrane Potential Detection kit (Mito Screen BD Biosciences). Fluorescence emission was analyzed by flow cytometry (JC-1 monomers, excitation wavelength $488 \mathrm{~nm}$, emission filter 530/30 nm; JC-1 aggregates, excitation wavelength $488 \mathrm{~nm}$, emission filter 585/42 nm) using a flow cytometer (FACSCalibur; Becton-Dickinson).

Statistical analysis. Statistical analyses were performed using the Student's t-test. All values are expressed as the means \pm standard error. P-values of $\leq 0.05$ were considered to indicate a statistically significant difference.

\section{Results}

PSK inhibits the proliferation of HL-60 cells. As shown in Fig. 1, PSK effectively inhibited cellular proliferation of HL-60 cells. PSK significantly inhibited cellular proliferation at $30 \mu \mathrm{g} / \mathrm{ml}$ in HL-60 cells.

PSK induces apoptosis. Next, we analyzed whether the growth inhibitory effect of PSK was attributable to the induction of apoptosis. To this end, we performed three different assays. As shown in Fig. 2, Annexin V-FITC and PI stained cells, which would be expected as late apoptotic or necrotic cells, were observed $48 \mathrm{~h}$ after PSK treatment and further increased until $72 \mathrm{~h}$ after treatment in HL-60 cells. 

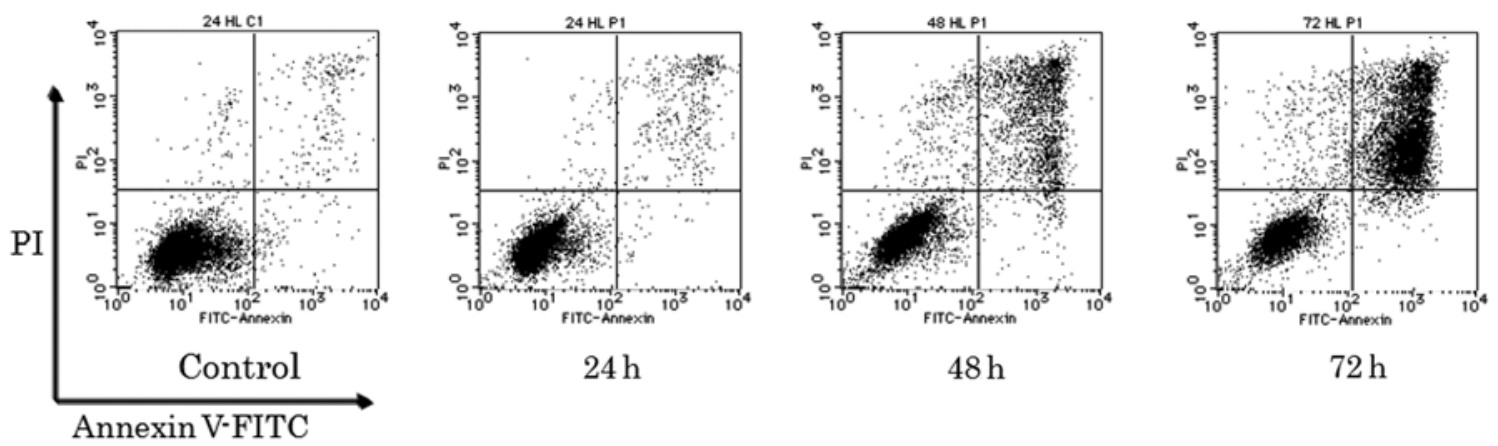

Figure 2. Effect of PSK on PI(+) Annexin V(+) staining. Representative PI and Annexin V profiles in HL-60 cells analyzed by flow cytometry following incubation with $30 \mu \mathrm{g} / \mathrm{ml}$ PSK. Control, cells incubated with DPBS.

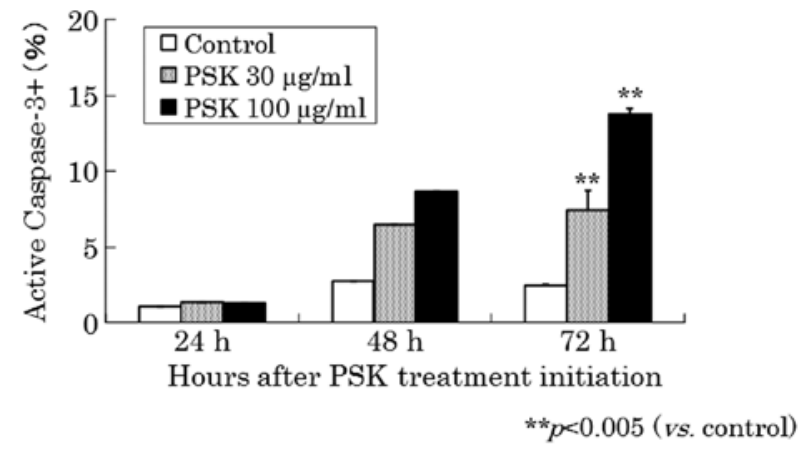

Figure 3. Effect of PSK on caspase-3 activation. The percentage of cells positive to active caspase-3 antibody was analyzed by flow cytometry. Each time-point represents the means \pm SD $(n=5)$. Statistical significance, ${ }^{* * *} \mathrm{P}<0.005$

PSK treatment activates caspase-3. To examine which proteins are involved in PSK-induced apoptosis, a comprehensive analysis of apoptosis-related proteins was performed. Proteome Profiler $^{\mathrm{TM}}$ Arrays Human Apoptosis array (R\&D Systems) indicated that several proteins were implicated in PSK-induced apoptosis (data not shown). Among these candidate proteins, we focused on caspase-3. As a result, active form of caspase-3 was detected $48 \mathrm{~h}$ after PSK treatment and further increased until $72 \mathrm{~h}$ after treatment (Fig. 3). These results suggested that caspase-3 activation is involved in PSK-induced apoptosis.

Phosphorylation of 38 MAPK. Several reports indicated that p38 MAPK plays an important role in apoptosis of HL-60 cells induced by various stimuli (16-19). Therefore, we examined the role of p38 MAPK in PSK-induced apoptosis. Firstly protein expression of the phosphorylated form of p38 MAPK, which is believed to be activated, was increased after $48 \mathrm{~h}$ of PSK treatment (Fig. 4).

Effect of p38 MAPK inhibitor on PSK-induced apoptosis. A p38 MAPK inhibitor, SB203580, was used to examine the role of p38 MAPK in PSK-induced apoptosis. PSK-induced apoptosis, as demonstrated by Annexin V-FITC and PI staining, was blocked by co-treatment with SB203580 (Fig. 5). The active form of caspase-3 was increased upon PSK treatment and was reduced by co-treatment with SB203580 (Fig. 6).

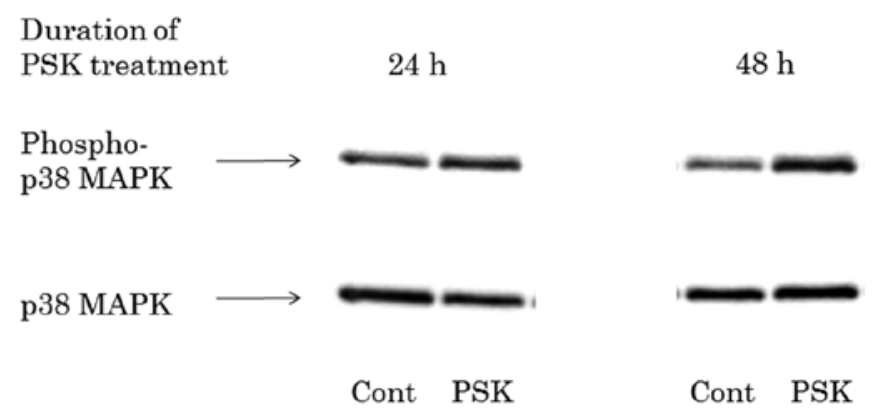

Figure 4. Effect of PSK on p38 MAPK phosphorylation in HL-60 cells After 24 or $48 \mathrm{~h}$ of incubation with DPBS (control) or PSK, cell lysates were obtained and subjected to western blotting. Data are representative of three independent experiments.

Furthermore, PSK-induced growth inhibition was also blocked by SB203580 (Fig. 7). These results suggest that p38 MAPK plays an important role in PSK-induced apoptosis and growth inhibition.

PSK induces mitochondrial pathway of apoptosis. To determine whether mitochondria were affected by PSK, we first examined the depolarization of $\Delta \Psi \mathrm{m}$ by measuring the fluorescence emission shift (red to green) of the $\Delta \Psi \mathrm{m}$ sensitive cationic JC-1 dye. JC-1 is readily taken up by mitochondria of healthy cells as a monomer. This uptake increases the concentration of the JC-1 inside the mitochondria leading to the formation of JC-1 aggregates which appear greenish red, whereas depolarized mitochondria do not accumulate JC-1 which remains in the cytoplasm as green colored control. Therefore, increase in green to red ratio symbolizes depolarization of mitochondria. PSK treatment showed a time-dependent increase in the green/red fluorescence intensity of HL-60 cells loaded with the JC-1 dye (Fig. 8).

Effect of p38 MAPK inhibitor SB203580 on PSK-induced mitochondrial pathway of apoptosis. A p38 MAPK inhibitor, SB203580, was used to examine the role of p38 MAPK in PSK-induced apoptosis. The depolarization of mitochondria induced by PSK treatment was reversed by co-treatment with SB203580 (Fig. 8). 
A

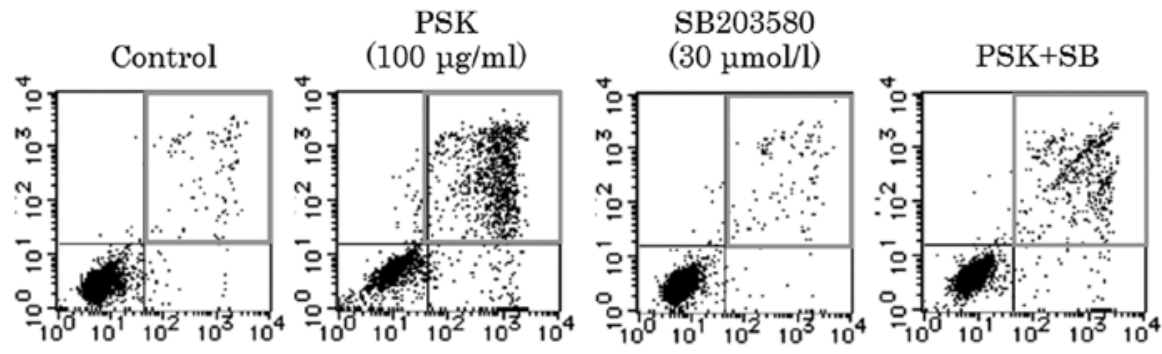

$48 \mathrm{~h}$
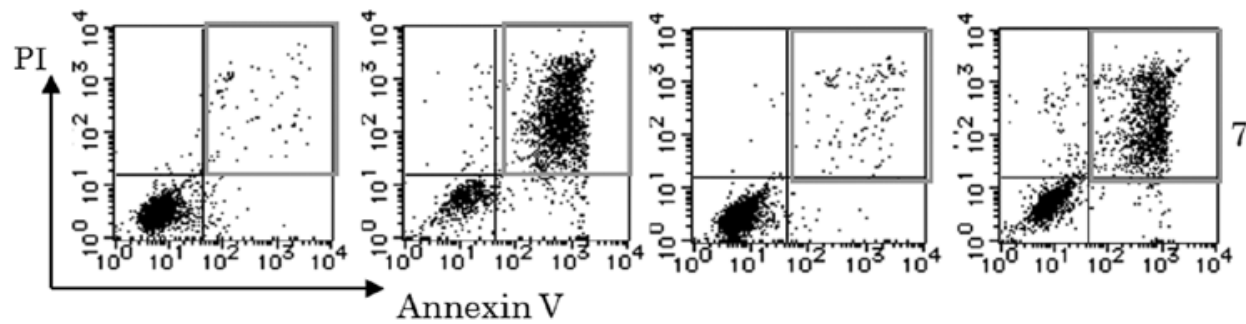

$72 \mathrm{~h}$

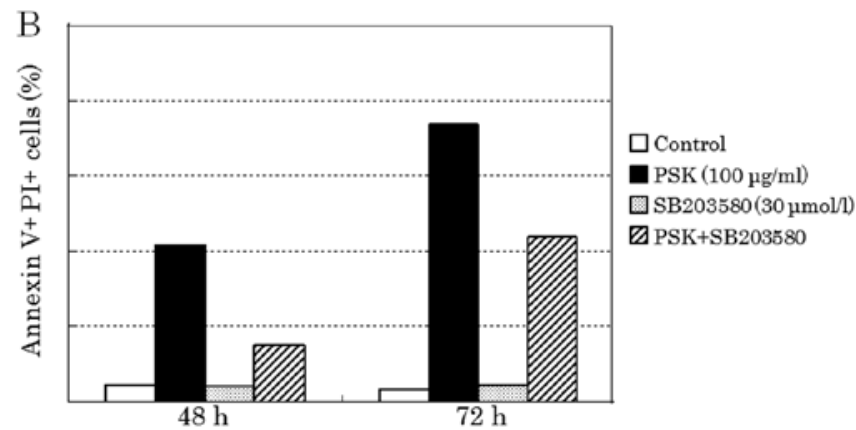

Figure 5. Effect of a p38 MAPK inhibitor, SB203580, on PSK-induced apoptosis. HL-60 cells were treated with DPBS (control) or PSK for 48 or $72 \mathrm{~h}$ in the presence or absence of SB203580. Cells were stained with fluorescein isothiocyanate (FITC)-conjugated Annexin V (Annexin V-FITC) and propidium iodide (PI), and analyzed by flow cytometry. (A) Flow-cytometric results; (B) data after numerical conversion. Data are representative of two independent experiments.

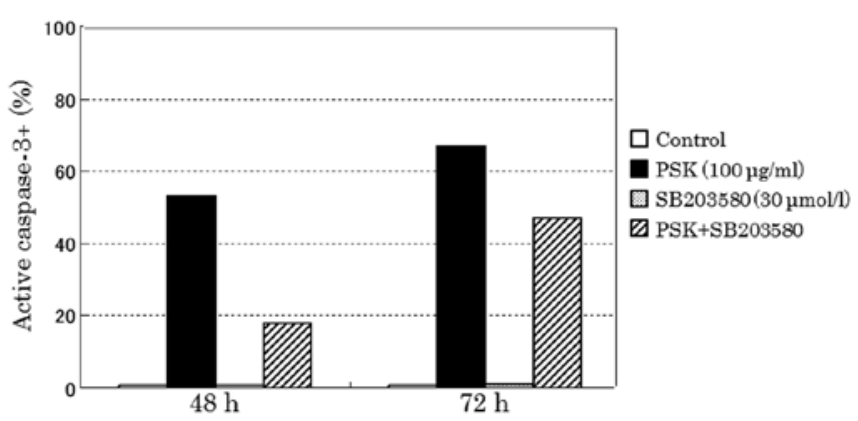

Figure 6. Effect of a p38 MAPK inhibitor, SB203580, on caspase-3 activation. HL-60 cells were treated with DPBS (control) or PSK for 48 or $72 \mathrm{~h}$ in the presence or absence of SB203580. Cells were stained with anti-active caspase- 3 antibody, and analyzed by flow cytometry. Data are representative of 2 independent experiments.

\section{Discussion}

To study the biological properties of protein-bound polysaccharide-K (PSK), we investigated the effects of PSK on cell proliferation in human leukemia HL-60 cells.

PSK-induced antiproliferative activity against HL-60 cells is caused, at least in part, by the induction of apoptosis,

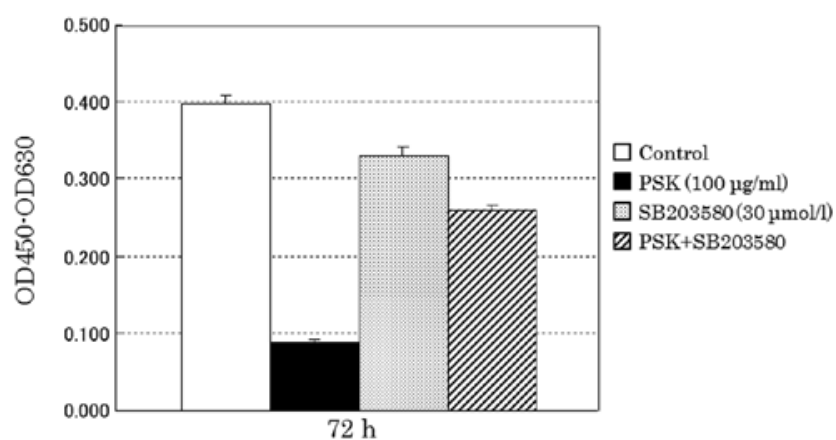

Figure 7. Effect of SB203580 on PSK-induced growth inhibition. HL-60 cells were treated with DPBS (control) or PSK for $72 \mathrm{~h}$ in the presence or absence of SB203580. Cellular proliferation was determined using WST-8, a watersoluble form of MTT. Data are expressed as the means $\pm \mathrm{SD}(\mathrm{n}=5)$. Data are representative of 2 independent experiments.

which has been reported, but has yet to be fully characterized (9-11). Comprehensive analysis of apoptosis-related protein expression upon PSK treatment of HL-60 cells showed altered expression of several proteins. Among these proteins, pro-caspase-3 was prominently upregulated. Pro-caspase- 3 is activated by various stresses and the active form of caspase- 3 plays a key role in the execution of apoptosis (3). We and 


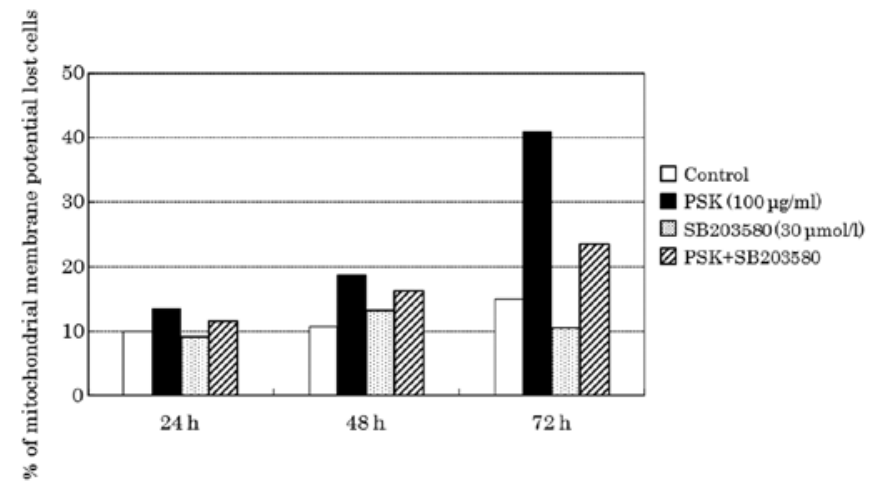

Figure 8. Effect of p38 MAPK inhibitor on PSK-induced loss of mitochondrial membrane potential. The mitochondrial membrane depolarization was induced by PSK in HL-60 cells, and the depolarization of mitochondria induced by PSK treatment was reversed by co-treatment with p38 MAPK inhibitor, SB203580.

other groups have found that the active form of caspase- 3 is increased upon PSK treatment (10). Therefore, caspase-3 plays an important role in PSK-induced apoptosis.

To assess the mechanism by which PSK inhibits HL-60 cell proliferation via induction of apoptosis, we focused on cell survival signaling in apoptosis. The MAPK signaling pathway played important roles in apoptosis induction. One important molecular mechanism for apoptosis might include p38- and Erk1/2-MAPK. We found altered expression of p38 MAPK protein in mitochondrion-dependent apoptosis.

MAPKs are a family of serine/threonine protein kinases that mediate signal transduction in mammals. MAPKs, which can be subdivided into ERK1/2, c-Jun N-terminal kinase (JNK), and p38 protein, are activated by modulation of important cellular functions, including proliferation, differentiation, or responses to environmental stimuli such as apoptosis (13). Apoptosis can be initiated via 2 alternative signaling pathways: the death receptor-mediated extrinsic apoptotic pathway and the mitochondrion-mediated intrinsic apoptotic pathway $(14,15)$. Mitochondria play a critical role in the regulation of various apoptotic processes, including drug-induced apoptosis. The mitochondrial apoptotic pathway is controlled by members of the Bcl-2 family, which decide cell fate via the interaction between pro- and antiapoptotic factors (16). The Bcl-2 family consists of pro- and antiapoptotic members (17).

In HL-60 cells, p38 MAPK is involved in the induction of apoptosis by various stimuli $(18,19)$. During apoptosis, p38 MAPK phosphorylates BCl-2 to inhibit its antiapoptotic properties, such as prevention of cytochrome $c$ release from the mitochondria $(20,21)$. The loss of $\Delta \Psi$ m may be a consequence of massive cytochrome $c$ release. Release of cytochrome $c$ from the mitochondria to the cytosol is a key event in mitochondrion-dependent apoptotic processes, leading to activation of caspase-9, which, in turn, activates caspase-3/7 (22). Park and Kim reported that auranofin induces p38 MAPK activation and apoptosis in HL-60 cells and that co-treatment with SB203580 prevents cytochrome $c$ release, caspase activation, and apoptosis (19). Therefore, PSK-induced p38 MAPK activation might trigger mitochondrion-dependent apoptotic processes. We observed the loss of $\Delta \Psi \mathrm{m}$ following PSK treatment; the p38 MAPK inhibitor SB203580 restored the PSK effect, suggesting the possibility that loss of $\Delta \Psi \mathrm{m}$ causes apoptotic cell death. However, we cannot rule out the possibility that loss of $\Delta \Psi \mathrm{m}$ is the result of apoptotic cell death, rather than its cause.

We have not examined the association between intracellular reactive oxygen species (ROS) and cytochrome $c$. Intracellular ROS act as secondary messengers in apoptosis induced by anticancer and chemopreventive agents (23). The generation of ROS can cause loss of $\Delta \Psi \mathrm{m}$ and induce apoptosis by releasing pro-apoptotic proteins such as apoptosis-inducing factor and cytochrome $c$ from the mitochondria to the cytosol. The generation of ROS may contribute to mitochondrial damage and may lead to cell death by acting as an apoptosis-signaling molecule (24).

In conclusion, PSK induces apoptosis in HL-60 cells through the activation of mitochondrial and caspase-dependent pathways involving activation of p38 MAPK signaling cascades. Other regulatory mechanisms that might be involved in PSK-induced signaling events remain to be identified in future studies.

\section{Acknowledgements}

This study was supported by Kureha Corporation, Tokyo, Japan.

\section{References}

1. Tsukagoshi S, Hashimoto Y, Fujii G, Kobayashi H, Nomoto K and Orita K: Krestin (PSK). Cancer Treat Rev 11: 131-155, 1984.

2. Nakazato H, Koike A, Saji S, Ogawa N and Sakamoto J: Efficacy of immunochemotherapy as adjuvant treatment after curative resection of gastric cancer. Lancet 343: 1122-1126, 1994.

3. Ohwada S, Ikeya T, Yokomori T, et al: Adjuvant immunochemotherapy with oral tegafur/uracil plus PSK in patients with stage II or III colorectal cancer: a randomized controlled study. Br J Cancer 90: 1003-1010, 2004.

4. Konno K, Motomiya M, Oizumi K, et al: Effects of proteinbound polysaccharide preparation (PSK) in small cell carcinoma of the lung. Haigan 28: 19-28, 1988.

5. Iguchi C, Nio Y, Takeda H, et al: Plant polysaccharide PSK: cytostatic effects on growth and invasion; modulating effect on the expression of HLA and adhesion molecules on human gastric and colonic tumor cell surface. Anticancer Res 21: 1007-1013, 2001.

6. Hirahara N, Fujioka M, Edamatsu T, et al: Protein-bound polysaccharide-K (PSK) induces apoptosis and inhibits proliferation of promyelocytic leukemia HL-60 cells. Anticancer Res 31: 2733-2738, 2011.

7. Evan GI and Vousden KH: Proliferation, cell cycle and apoptosis in cancer. Nature 411: 342-348, 2003.

8. Hu W and Kavanagh JJ: Anticancer therapy targeting the apoptosis pathway. Lancet Oncol 4: 721-729, 2003.

9. Yefenof E, Gafanovitch I, Oron E, Bar M and Klein E: Prophylactic intervention in radiation-leukemia-virus-induced murine lymphoma by the biological response modifier polysaccharide K. Cancer Immunol Immunother 41: 389-396, 1995.

10. Hattori ST, Komatsu N, Shichijo S and Itoh K: Protein-bound polysaccharide $\mathrm{K}$ induced apoptosis of the human Burkitt lymphoma cell line, Namalwa. Biomed Pharmacother 58: 226-230, 2004.

11. Jiménez-Medina E, Berruguilla E, Romero I, et al: The immunomodulator PSK induces in vitro cytotoxic activity in tumor cell lines via arrest of cell cycle and induction of apoptosis. BMC Cancer 8: 78, 2008.

12. Thornberry NA: Caspases: key mediators of apoptosis. Chem Biol 5: R97-R103, 1998.

13. Chen X, Lan X, Mo S, et al: p38 and ERK, but not JNK, are involved in copper-induced apoptosis in cultured cerebellar granule neurons. Biochem Res Commun 379: 944-948, 2009. 
14. Reuter S, Eifes S, Dicato M, Aggarwal BB and Diederich M: Modulation of anti-apoptotic and survival pathways by curcumin as a strategy to induce apoptosis in cancer cells. Biochem Pharmacol 76: 1340-1351, 2008.

15. Cory S and Adams JM: The Bcl-2 family: regulators of the cellular life-or-death switch. Nat Res Cancer 2: 647-656, 2002.

16. Leibowitz B and Yu J: Mitochondrial signaling in cell death via the Bcl-2 family. Cancer Biol Ther 9: 417-422, 2010.

17. Xiao G, Fang $\mathrm{H}$, Xing $\mathrm{C}$ and $\mathrm{Xu}$ W: Structure, function and inhibition of Bcl-2 family proteins: a new target for anti-tumor agents. Mini Rev Med Chem 9: 1596-1604, 2009.

18. Ahn YH, Jung JM and Hong SH: 8-chloro-cyclic AMP-induced growth inhibition and apoptosis is mediated by p38 mitogenactivated protein kinase activation in HL60 cells. Cancer Res 65 4896-4901, 2005.

19. Park SJ and Kim IS: The role of p38 MAPK activation in auranofin-induced apoptosis of human promyelocytic leukemia HL-60 cells. Br J Pharmacol 146: 506-513, 2005.
20. Torcia M, De Chiara G, Nencioni L, et al: Nerve growth factor inhibits apoptosis in memory B lymphocytes via inactivation of p38 MAPK, prevention of BCL-2 phosphorylation, and cytochrome release. J Biol Chem 276: 39027-39036, 2001.

21. De Chiara G, Marcocci ME, Torcia M, et al: BCL-2 phosphorylation by p38 MAPK. J Biol Chem 281: 21353-21361, 2006.

22. Riedl SJ and Salvesen GS: The apoptosome: signalling platform of cell death. Nat Rev Mol Cell Biol 8: 405-413, 2007.

23. Garcia A, Morales P, Arranz N, Delgado ME, Rafter J and Haza AI: Antiapoptotic effects of dietary antioxidants towards $\mathrm{N}$-nitrosopiperidine and $\mathrm{N}$-nitrosodibutylamine-induced apoptosis in HL-60 and HepG2 cells. J Appl Toxicol 29: 403-413, 2009.

24. Zhang R, Humphreys I, Sahu RP, Shi Y and Srivastava SK: In vitro and in vivo induction of apoptosis by capsaicin in pancreatic cancer cells is mediated through ROS generation and mitochondrial death pathway. Apoptosis 13: 1465-1478, 2008. 